\title{
Cathodoluminescence Spectra Acquisition Using an Imaging Spectrograph and CCD Detector: Materials Characterization Using Trivalent REE Doped Synthetic and Natural Materials
}

\author{
J.M. Hanchar ${ }^{1}$ \\ ${ }^{1}$ Memorial University of Newfoundland, St. John's, Canada
}

Catholuminescence (CL) is a powerful imaging and spectroscopic technique that can be used to study a wide range of materials. This includes natural and synthetic minerals, and other types of ceramic materials, that contain either naturally occurring, or intentionally doped, transition metals, rare earth elements (REE), or the uranyl ion [1], respectively. Cathodoluminescence imaging can be used in natural and synthetic minerals to reveal internal zoning (growth and sectoral), radiation damage in different regions in crystals, and in some cases can be used to reveal different geologic events (Fig. 1). Often these features are invisible or barely visible using transmitted or reflected light microscopy. Cathodoluminescence spectroscopy can often be used to identify the element(s) causing the CL emission and with sufficient spectral resolution (e.g., greater than $1 \mathrm{~nm}$ ) quantify the crystal field effects of the impurity ion by the host material.

A custom-built petrographic microscope-based CL imaging and spectroscopy system that utilizes an imaging spectrograph-CCD (charge-coupled device) detector and high-end digital camera, will be discussed. This system has several unique advantages for acquiring high-resolution CL images and emission spectra in luminescing materials: 1) high spatial resolution with the ability to accurately collect CL images and spectra from regions as small as 10 by 10 micrometers in materials; 2) high spectral resolution of $\sim 0.08-0.15 \mathrm{~nm}$ (depending on focal length of the spectrometer and the groove density of the diffraction grating used); 3) by being interfaced to a petrographic microscope the colour of the CL emission can be accurately calibrated which is an advantage over SEM or EPMA based CL systems; and 4) This system allows for the acquisition of highresolution CL spectra in bulk specimens of appropriate thickness as well as isolated regions in mineral grains in thin sections or in epoxy mounts.

Applications using this CL imaging and spectroscopy system will be presented with examples of materials that includes natural zircon (Fig. 1), synthetic zircon $\left(\mathrm{ZrSiO}_{4}\right)$ doped with individual rare earth elements (Fig. 2), synthetic titanite $\left(\mathrm{CaTiSiO}_{5}\right)$ glass and crystals (Fig. 3), and other ceramic and geological materials doped with $\mathrm{Dy}^{3+}$ (Fig. 3). Although it is commonly assumed that the crystal field of the host material exerts little influence on the REE $4 \mathrm{f}$ electrons, we demonstrate that this is not the case and that each of the Dy-doped materials has a unique CL emission spectrum. In order to measure these differences a high-resolution imaging and spectroscopy system is required in order to allow for images and CL emission spectra to be acquired from selected regions of interest. 


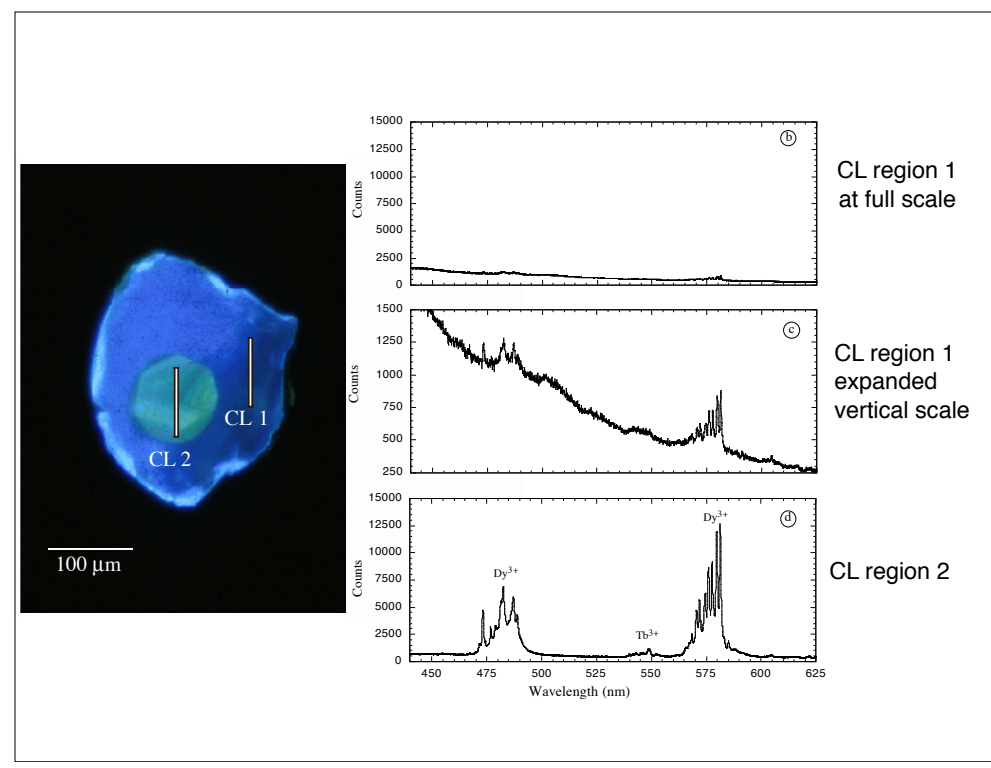

Figure 1. CL image (left) and spectra from igneous core and metamorphic overgrowth of a natural zircon crystal. (a) blue CL rim region 1 at same vertical scale as (c). (b) blue CL region 1 with vertical scale expanded 10x, and ( $\mathrm{f}$ ) green CL core region 2 at same vertical scale as (a) [3].

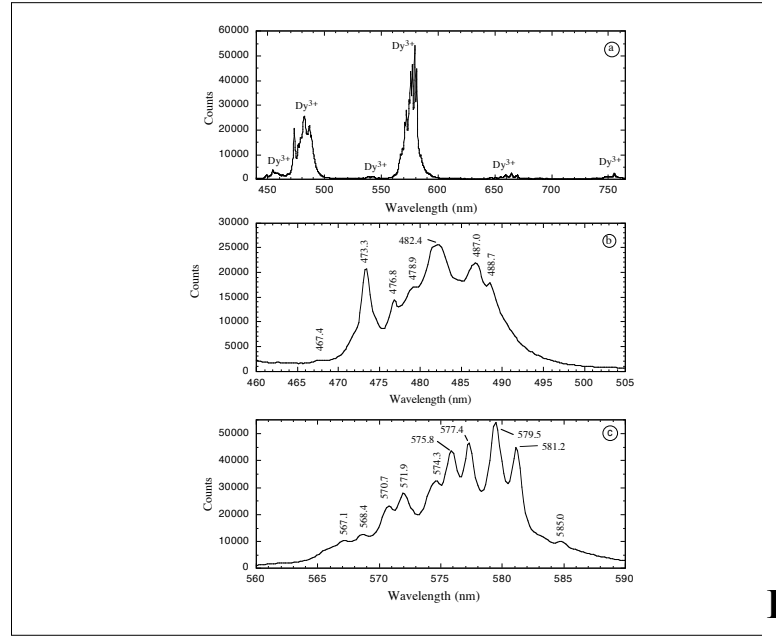

Figure 2. Cathodoluminescence spectra of synthetic $\mathrm{Dy}^{3+}$ doped zircon crystal. Full spectrum (a), and zoom of main $4 \mathrm{f}$ transition regions at $\sim 480$ $\mathrm{nm}(\mathrm{b})$ and $\sim 575 \mathrm{~nm}(\mathrm{c})$.

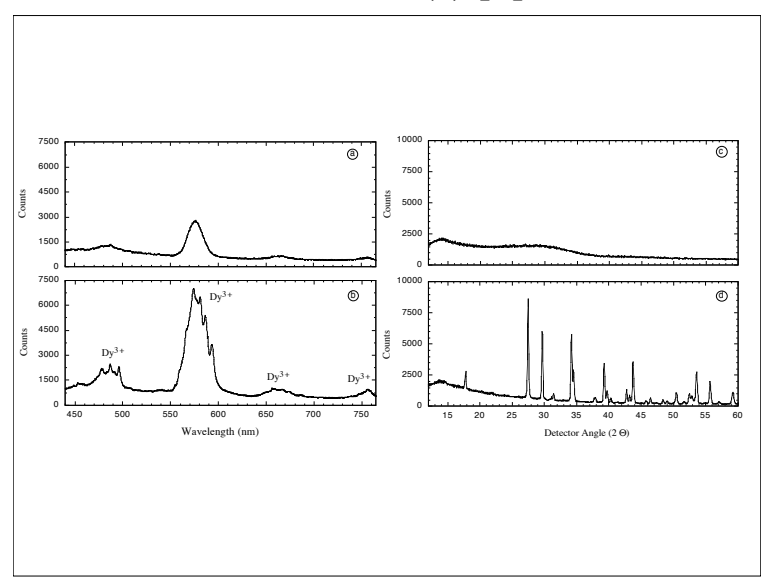

Figure 3. Cathodoluminescence spectra of synthetic $\mathrm{Dy}^{3+}$ doped titanite glass (a) and crystals (b), and powder X-ray diffraction scan of same glass (c) and crystals (d).

\section{References:}

[1] BG Yacobi and DB Holt (1990) "Cathodoluminescence Microscopy of Inorganic Solids", (Plenum, New York) $292 \mathrm{p}$.

[2] JM Hanchar and CF Miller, Chemical Geology, 110 (1993), pp. 1-13. [3] JM Hanchar and RL Rudnick, Lithos, 36 (1995), pp. 289-303. 